\title{
Self-Dual Chern-Simons Solitons and Quantum Potential
}

Oktay K PASHAEV ${ }^{\dagger_{1}, \dagger_{2}}$ and Jyh-Hao LEE $\ddagger$

$\dagger_{1}$ Joint Institute for Nuclear Research, Dubna (Moscow), 141980, Russian Federation E-mail: pashaev@vxjinr.jinr.ru

$\dagger_{2}$ Department of Mathematics, Izmir Institute of Technology Cankaya-Izmir, Turkey E-mail: pashaev@likya.iyte.edu.tr

$\ddagger$ Institute of Mathematics, Academia Sinica, Taipei, 11529, Taiwan

E-mail: leejh@ccvax.sinica.edu.tw

\begin{abstract}
An influence of the quantum potential on the Chern-Simons solitons leads to quantization of the statistical parameter $\kappa=m e^{2} / g$, and the quantum potential strenght $s=1-m^{2}$. A new type of exponentially localized Chern-Simons solitons for the Bloch electrons near the hyperbolic energy band boundary are found.
\end{abstract}

\section{Introduction}

Extensions of the Schrödinger equation by the quantum potential non-linearity have been considered in connection with several problems $[1,2,3]$ :

a) to allow formally the diffusion coefficient of the stochastic process in a stochastic quantization to differ from $\hbar / 2 m$, related to the difference in the Plank constant or the inertial mass;

b) to allow corrections from quantum gravity. Below we find exactly solvable cases for $2+1$ dimensional nonlinear Schrödinger (NLS) model interacting with ChernSimons field under the influence of the quantum potential, which could represent the stochastically quantized anyons.

\section{Self-dual solitons hierarchy}

The Lagrangian of the modified Jackiw-Pi model [4]:

$$
L=\frac{\kappa}{2} \epsilon^{\mu \nu \lambda} A_{\mu} \partial_{\nu} A_{\lambda}+\frac{i}{2}\left(\bar{\psi} D_{0} \psi-\psi \bar{D}_{0} \bar{\psi}\right)-\overline{\mathbf{D}} \bar{\psi} \mathbf{D} \psi+s \nabla|\psi| \nabla|\psi|+g|\psi|^{4},
$$

where $D_{\mu}=\partial_{\mu}+i e A_{\mu}(\mu=0,1,2)$, includes an additional quantum potential term with strenght $s$. In classical equations of motion for $s<1$ we decompose $\psi=e^{R-i S}$ and introduce new rescaled variables $t=\tilde{t} / \sqrt{1-s}, S=\sqrt{1-s} \tilde{S}, A_{0}=(1-s) \tilde{A}_{0}$, 
$\mathbf{A}=\sqrt{1-s} \tilde{\mathbf{A}}$. Then for the new function $\tilde{\psi}=\exp (R-i \tilde{S})$, we get the standard gauged NLS

$$
i \tilde{D}_{\tilde{0}} \tilde{\psi}+\tilde{\mathbf{D}}^{2} \tilde{\psi}+\frac{2 g}{1-s}|\tilde{\psi}|^{2} \tilde{\psi}=0
$$

but with rescaled $\tilde{\kappa}=\kappa(1-s)^{1 / 2}, \tilde{g}=g /(1-s)$. When the coupling constants are restricted by the condition $g \kappa / e^{2}=\sqrt{1-s}$ the static solutions require the self-dual Chern-Simons equations with modified magnetic flux in the Gauss law

$$
\tilde{D}_{-} \tilde{\psi}=0, \quad \partial_{1} \tilde{A}_{2}-\partial_{2} \tilde{A}_{1}=\frac{e}{\kappa(1-s)^{1 / 2}} \tilde{\bar{\psi}} \tilde{\psi}
$$

Inserting $\mathbf{A}$ from the first equation into the second one we get the Liouville equation with radially symmetric solutions

$$
\rho(r)=4 \frac{\kappa(1-s)^{1 / 2} N^{2}}{e^{2} r^{2}}\left[\left(\frac{r}{r_{0}}\right)^{N}+\left(\frac{r_{0}}{r}\right)^{N}\right]^{-2},
$$

which would be regular if $N \geq 1$. Then, from the regularity of the gauge potential $\mathbf{A}$ we can fix the phase of $\tilde{\psi}=\exp (R-i \tilde{S})$ as $\tilde{S}=(N-1) \theta, \theta=\arctan x_{2} / x_{1}$, and restrict $N$ to be an integer for single-valued $\tilde{\psi}$. However, the auxiliary function $\tilde{\psi}$ is not the physical one, this is the reason that fixing an integer $N$ is not sufficient for single-valuedness of the original function $\psi=\exp \left(R-i(1-s)^{1 / 2}(1-N) \theta\right)$. An integer valued must be the product $(1-s)^{1 / 2}(N-1)=n$, which requires an integer-valuedness of $\sqrt{1-s}=m$ and as the consequence of (5), we obtain the quantization condition $\frac{g \kappa^{2}}{e^{2}}=m,(m=1,2,3, \ldots)$. The latter means that in the presence of quartic self-interaction the Chern-Simons coupling constant and the quantum potential strength must be quantized

$$
\kappa=m \frac{e^{2}}{g}, \quad s=1-m^{2} .
$$

In this relation the $g$ and $\kappa$ coupling constants play a role similar to the one in the electro-magnetic duality condition derived by Dirac. The self-dual system (3) for original $(\psi, \mathbf{A})$ has a form depending on the quantum potential with a coefficient proportional to the angular defect, and turn the energy to vanish $H=0$. This shows that under the influence of the quantum potential Chern-Simons solitons continue to be a zero energy configuration. Due to (5) the flux for solution (4) is quantized $\Phi=\frac{2 \pi}{e}(2 m) N$, where $N=1,2,3, \ldots$ and the value of $m$ is fixed. Thus, the magnetic flux of our vortex/soliton is an even multiple $m$ of the elementary flux quantum. This generalizes the Jackiw-Pi result related to the particular value $m=1$.

The decomposition $\psi=\exp (R-i S)$ in quantum mechanics produces the Madelung fluid representation. For the self-dual flows, with velocity $\mathbf{v}=-2(\nabla S-e \mathbf{A})$ we find the canonical Hamiltonian equations

$$
\dot{x} \equiv v_{1}=\frac{\partial \chi}{\partial y}, \quad \dot{y} \equiv v_{2}=-\frac{\partial \chi}{\partial x},
$$

where the stream function $\chi$, plays the role of a Hamiltonian, $\chi=(1-s)^{1 / 2} \ln \rho$. For soliton (4), $\rho$ is regular everywhere and has $2(N-1)$-th order zero at the origin of the coordinates. This zero determines a singularity of $\chi$ and $\mathbf{v}$, such that near the 
singularity $\chi=2(N-1)(1-s)^{1 / 2} \ln r \equiv-\frac{\beta}{2 \pi} \ln r$, corresponding to the line vortex, with quantized strength $\beta=-4 \pi n=4 \pi m(1-N)$. The velocity field near the center $\mathbf{v}=\left(-2 n / r^{2}\right)(-y, x)$, is the gradient of multivalued function $\phi=-2 n \arctan (y / x)$, which fixes the value of the phase of the wave function $\psi$. Functions $\chi$ and $\phi$ are conjugate harmonic functions, providing holomorphicity condition for the gauge potential near the center. This shows that Chern-Simons soliton can be interpreted as a planar vortex in the Madelung fluid, having the form of a linear vortex near the rotation point.

\section{Exponentially localized Chern-Simons solitons}

In the dynamics of Bloch electron in a solid for two-dimensional motion, in simplest case of the energy surface near the band boundary $E(\mathbf{k})=k_{1}^{2} / 2 m-k_{2}^{2} / 2 m$, the effective mass matrix is $M^{-1}=\operatorname{diag}(1 / m,-1 / m)$ and the Bloch electron subject to the Lorentz force, imitated by Chern-Simons interaction, has a "dissipative" character [5], with the constant magnetic field playing the role of the damping. Then, in Lagrangian (1) and equations of motion (2) we replace the positive space metric $\eta_{i j}=\operatorname{diag}(1,1)$ with the indefinite one $\eta_{i j}=\operatorname{diag}(1,-1)$ so that $\partial_{a} \partial^{a}=\eta^{a b} \partial_{a} \partial_{b}=\partial_{1}^{2}-\partial_{2}^{2}$. Now we rescale $t=\tilde{t} / \sqrt{s-1}$, $S=\sqrt{s-1} \tilde{S}, A_{0}=(s-1) \tilde{A}_{0}, \mathbf{A}=(s-1)^{1 / 2} \tilde{\mathbf{A}}$ and instead of $\psi=\exp (R-i S)$, introduce two real functions $Q^{ \pm}=\exp (R \pm \tilde{S})$, satisfying the gauged pair of time-reversal invariant diffusion equations with the reaction term $Q^{+} Q^{-}$:

$$
\mp \tilde{D}_{\tilde{0}}^{\mp} Q^{ \pm}+\tilde{D}_{a}^{\mp} \tilde{D}^{a \mp} Q^{ \pm}-\frac{2 g}{s-1} Q^{+} Q^{-} Q^{ \pm}=0,
$$

where $\tilde{D}_{\mu}^{ \pm}=\partial_{\mu} \pm e \tilde{A}_{\mu}$ and instead of the local $U(1)$ phase transformations now ChernSimons gauge field corresponds to the local rescaling of $Q^{ \pm}$. For static configurations, when $\sqrt{s-1}=-g \kappa / e^{2}$ we have the self-duality equations

$$
\tilde{D}_{-}^{-} Q^{+}=0=\tilde{D}_{+}^{+} Q^{-}, \quad\left[\tilde{D}_{+}^{ \pm}, \tilde{D}_{-}^{ \pm}\right]=\mp \frac{2 e^{2}}{\kappa(s-1)^{1 / 2}} Q^{+} Q^{-},
$$

with $\tilde{D}_{ \pm}^{a}=\tilde{D}_{1}^{a} \pm \tilde{D}_{2}^{a}$, from which for $\rho=Q^{+} Q^{-}$we obtain the Liouville equation

$$
\left(\partial_{1}^{2}-\partial_{2}^{2}\right) \ln \rho=2 \frac{e^{2}}{\kappa(s-1)^{1 / 2}} \rho .
$$

admitting the general solution in terms of two arbitrary functions $A\left(x_{+}\right)$and $B\left(x_{-}\right)$of $x_{ \pm}=x_{1} \pm x_{2}$. The known regular soliton solutions lead to the divergent integral for $\rho$, thus they have no physical meaning in our problem. If we choose instead the $A$ and $B$ functions in the form

$$
A=(a+1) \operatorname{coth}^{2 k+1} \frac{\alpha}{2} x_{+}, \quad B=(a-1) \tanh ^{2 l+1} \frac{\beta}{2} x_{-},
$$

then we get

$$
\rho \sim \frac{\left(1-a^{2}\right) \alpha \beta \sinh ^{2 k} \alpha x_{+} \sinh ^{2 l} \beta x_{-}}{\left[(a+1) \cosh ^{2 k+1} \frac{\alpha x_{+}}{2} \cosh ^{2 l+1} \frac{\beta x_{-}}{2}+(a-1) \sinh ^{2 k+1} \frac{\alpha x_{+}}{2} \sinh ^{2 l+1} \frac{\beta x_{-}}{2}\right]^{2}} .
$$

For $a>0$ this solution is nonsingular in the whole $\left(x_{1}, x_{2}\right)$ plane. The sign definiteness of $\rho$ requires $k, l$ to be integer, while regularity at the "light-cone", $x_{+}=0$ or $x_{-}=0$ is valid 
for $k \geq 0, l \geq 0$. In contrast with the algebraic Chern-Simons solitons (4), the solution is exponentially decreasing in the plane for the "future" and "past" null infinities. It is worth to note that our solutions are related with exponentially localized planar solitons $\left(E L_{2}\right)$ for the Davey-Stewartson-I (DS-I) equation. Comparing to (4), having for $N>1$ the zero at the origin of the coordinates, the zeroes for solution (9) $\rho \sim x_{+}^{2 k} x_{-}^{2 l}$ are located along the "light cone" $x_{+}=0, x_{-}=0$ for $k>0$ and $l>0$ correspondingly. For $k=0$ and $l=0$, we have exponentially localized soliton located at the beginning of coordinates. If only one of the $k, l$ values is vanishing, then we have two soliton solution represented on Fig. 1a. and symmetrical under one of the light cone directions. When both numbers are nonzero we get a four-soliton solution, represented on Fig. 1b, with zeroes along the whole light cone. For solution (9), the singularities of potential A are located along the light cone and can be compensated by derivation of function $\tilde{S}$ as $\tilde{S}=\ln \left|x_{+}^{k} / x_{-}^{l}\right|$. This allows us to define exact solutions of Eqs. (7) and the original system as

$$
\psi=\sqrt{\rho}\left(\frac{x_{-}^{l}}{x_{+}^{k}}\right)^{i \sqrt{s-1}} .
$$

For particular values $k=l$, the phase $\tilde{S}=k \ln \left|x_{+} / x_{-}\right|$defines the hyperbolic analog of the singular Aharonov-Bohm potential $e a_{i}=2 k \epsilon_{i j} x_{j} /\left(x_{1}^{2}-x_{2}^{2}\right)$ having singularities not only at the origin of the coordinates, but also along the "light-cone", $x_{+}=0, x_{-}=0$. It may be represented as a singular pure gauge $e a_{i}=-\partial_{i} \tilde{S}=-2 k \partial_{i} \theta$, where $\tilde{S}=2 k \theta$. The magnetic flux associated with soliton solutions is independent of $k$ and $l$, and is not quantized. Moreover, the phase of $\psi$ includes the hyperbolic rotation angle $\theta$ which is valued on the whole real line and no restriction of single-valuednees arises.

\section{Conclusions}

Our hyperbolic self-duality equations are equivalent to $S O(2,1) / O(1,1)$ self-dual $\sigma$ model and our vortex configurations (9) for $a>0$ generate regular non-topological solution. For topologically nontrivial configurations we need to consider $a<0$, which would produce singularity for $\rho$.

In conclusions we stress that two long-range interactions considered in the present paper, the Chern-Simons gauge interaction introduced to physics by S Deser, R Jackiw and $\mathrm{S}$ Templeton and the quantum potential introduced by $\mathrm{L}$ de Broglie and D Bohm, are compatible in supporting static soliton solutions in $2+1$ dimensions, with arbitrary $N$, only when the coupling constants for both interactions are quantized. 

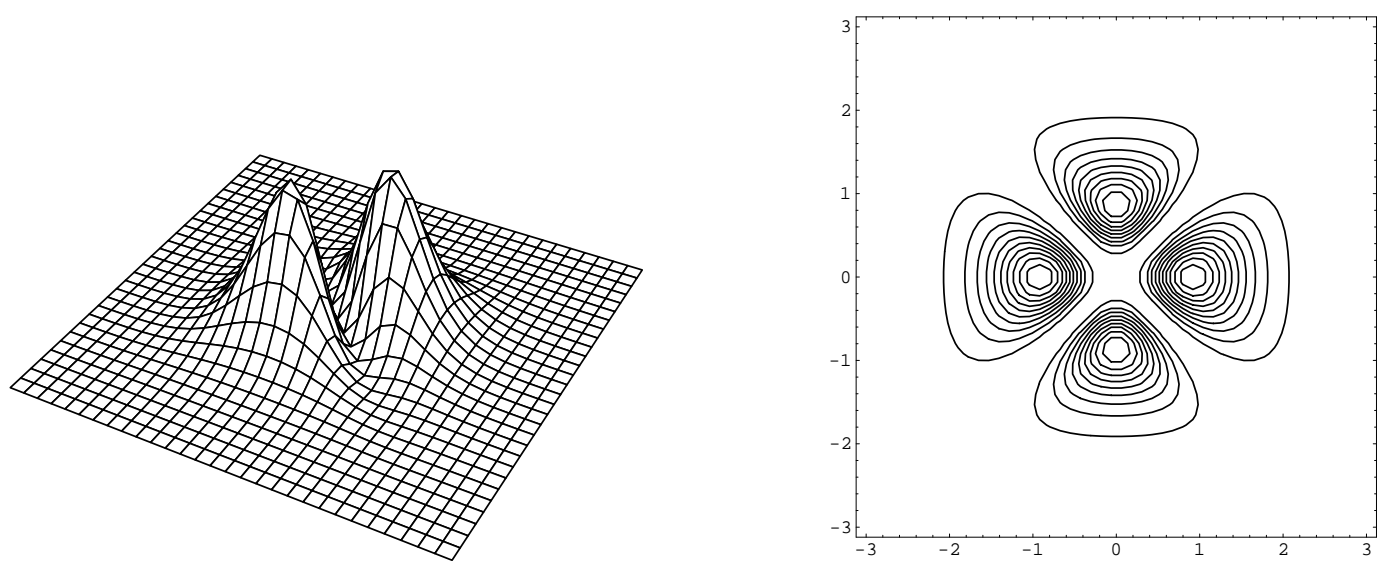

Figure 1. a) 3D plot of two Chern-Simons solitons; b) Contour plot of four solitons.

\section{References}

[1] Guerra F and Pusterla M, Lett. Nuovo Cimento, 1982, V.34, 351;

Smolin L, Phys. Lett. A, 1986, V.113, 1986;

Bertolami O, Phys. Lett. A, 1991, V.154, 1991.

[2] Vigier J-P, Phys. Lett. B, 1989, V.135, 99;

Doebner H-D, Goldin G A and Nattermann P, J. Math. Phys., 1999, V.40, 49.

[3] Sabatier P C, Inverse Problems, 1990, V.6, L47;

Auberson G and Sabatier P C, J. Math. Phys., 1994, V.35, 4028.

[4] Jackiw R and Pi S-Y, Phys. Rev. Lett., 1990, V.64, 2969.

[5] Blazone M, Graziano E, Pashaev O K and Vitiello G, Annals of Physics (NY), 1996, V.252, 115. 